\title{
Pressure Measurement by Medical Devices and within Medical Devices
}

Glocker, Raymond

MHM Harzbecher Medizintechnik GmbH

Erthalstr. 12, 63739 Aschaffenburg

There exist lots of historical pressure units as bar, atm., mmWs, but allowed today in $\mathrm{SI}=$ Système International d'unités are only Pascal, Newton $/ \mathrm{m}^{2}$, exception for medicine: also the pressure unit $\mathrm{mm} \mathrm{Hg}$ is allowed.

A further difference which will show up in medical applications shall be mentioned: the word "monitoring" means in technical applications: to observe a situation for any changes which may occur over time. In medicine, it also means that, but in addition medical monitoring means: plus alarm. The standard for patient monitors is: ISO 606012-49.

Pressures in the human body occur in fast changes and in slow changes:
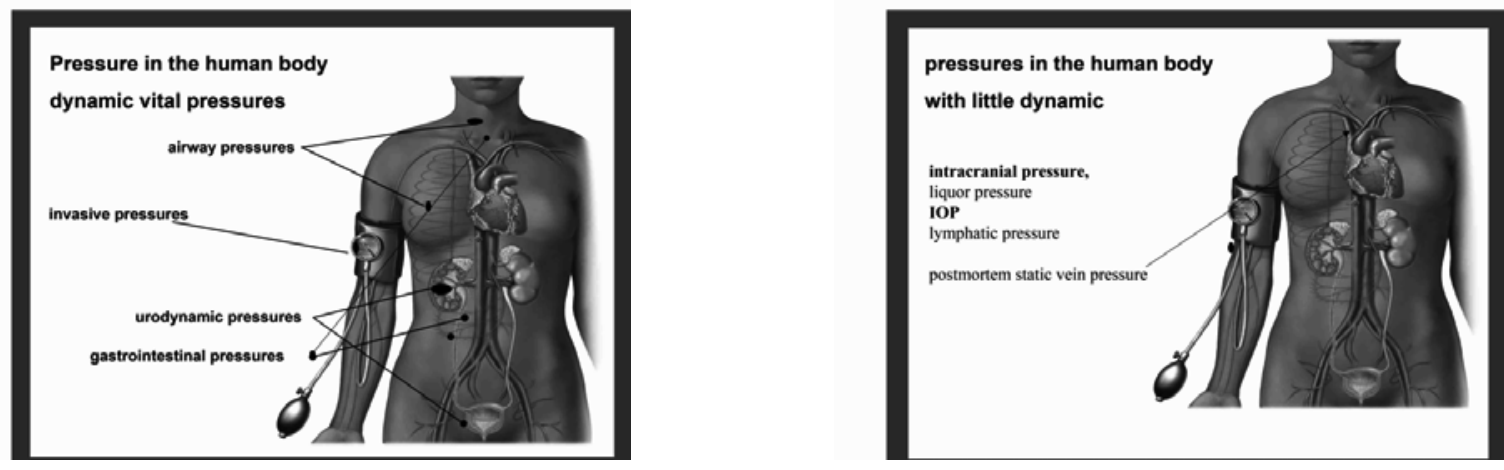

What everybody knows as it belongs to our normal experience: cuff-measurement of our arterial blood pressure, which is explained by the following graphic:

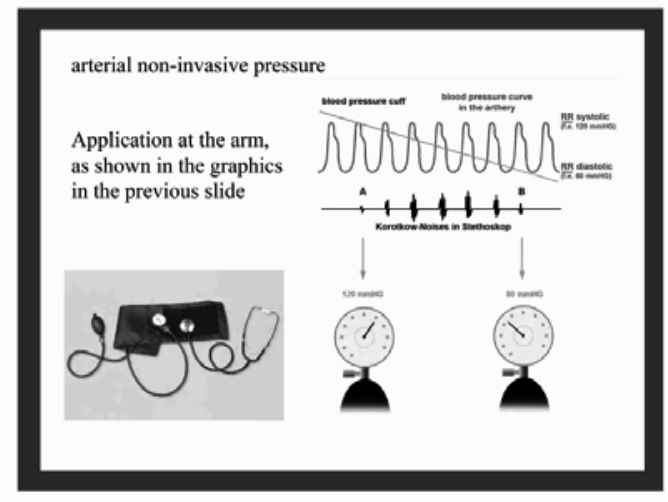

The real dynamic of the pressure curve in safe and sound conditions between $80 \mathrm{~mm}$ $\mathrm{Hg}$ (diastolic value) and $120 \mathrm{~mm} \mathrm{Hg}$ (systolic value) cannot be evaluated by this method, as the acoustic observation of pulse wave sound to start and to end is no recording of the pressure curve. If the patient is hospitalised and there is an arterial access (mostly at the radial artery), then the pressure curve can be recorded in full with a transducer (will be explained later), transferring the mechanical pressure signal to an electrical signal

And the same applies to other blood pressures, most common venous pressure (smaller pressure values around $10 \mathrm{~mm} \mathrm{Hg}$ ) and the pulmonary artery pressure. 

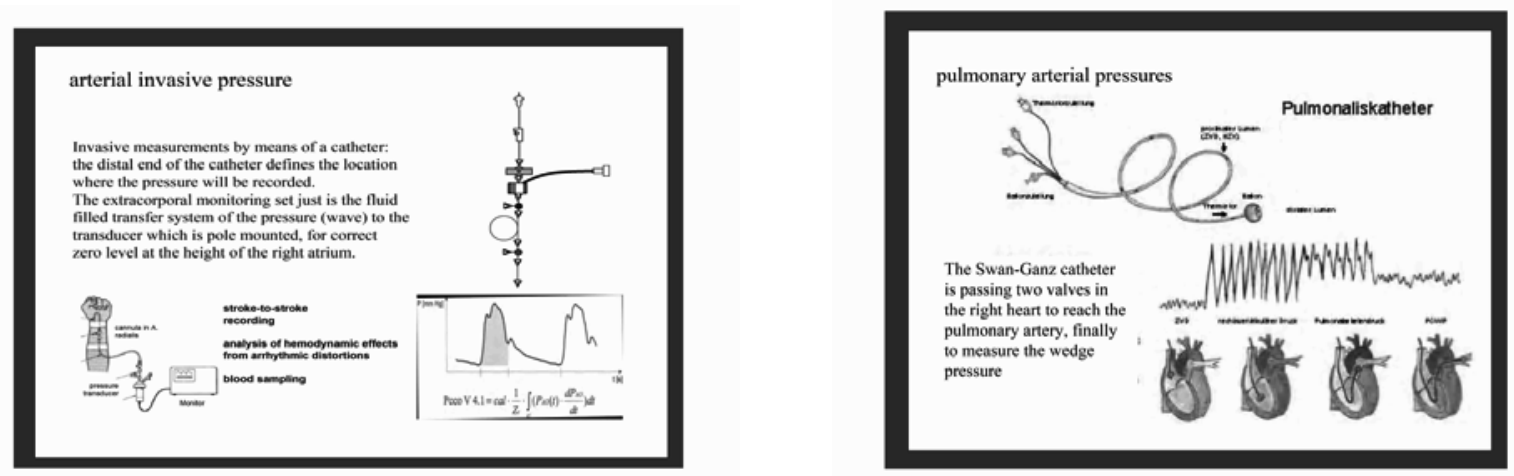

Airway pressure measurements are used in wide variations at these many ventilation procedures (listed alphabetically):

APRV (airway pressure release v.), ASB (assisted spontaneous breathing), ASV (adaparive support v.), ATC (automatic tube compensation), BIPAP (biphasic positive airway pressure), CMV (continuous mandatory v.), CPAP (continuous positive airway pressure), CPPV (continuous positive pressure v.), EPAP (expiratory positive airway pressure), HFPPV (high frequency positive pressure v.), HFOV (high frequency oscillatory v.), ILV (independent lung v.), IPAP (inspuiratory positive airway pressure), IPPV (intermittend positive pressure v.), IRV (inversed ratio v.), LFPPV (low frequency positive pressure v.), MMV (mandatory minute volume), MPAV (proportional assist v.), PCMV 8pressure controlled mandatory v.), PCV (pressure controlled v.), PEEP (positive endexpiratory pressure), PNPV ((positive negative pressure v.), S-CPPV (synchronised continuous positive pressure v.), S-IPPV (as before, intermittend instead of continuous), (S)IMV (synchronisedintermittend mandatory v.), VCMV (volume controlled mandatory v.), VCV (volume controlled v.)

Urodynamic pressures will be measured on special chair equipment, as also the socalled gastro-intestinal manometry. Intracranial pressure (ICP) has nearly no dynamics,

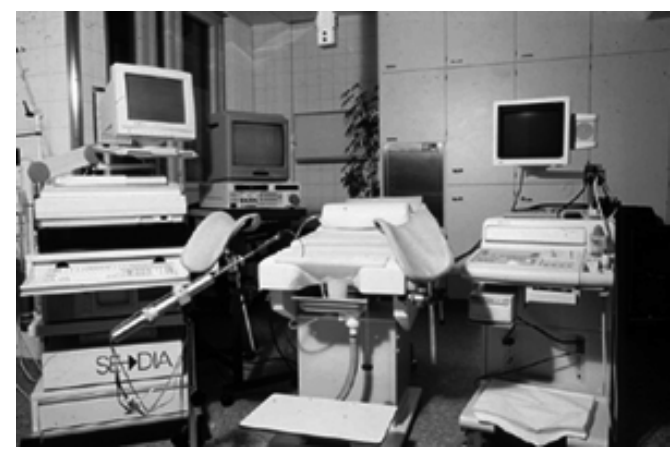

IOP, intra ocular pressure main 3 Methods

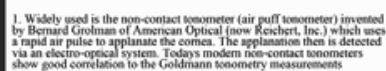

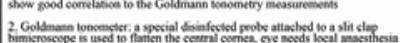

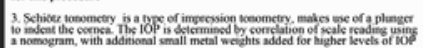

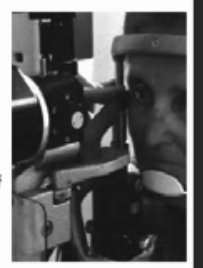
therefore even an air filled connection to the transducer is satisfactory, much more important than the pressure sensor is hygienic protection.

Normal ICP: $5-15 \mathrm{mmHg}$, during coughing 50-80 $\mathrm{mmHg}$ can b.e achieved

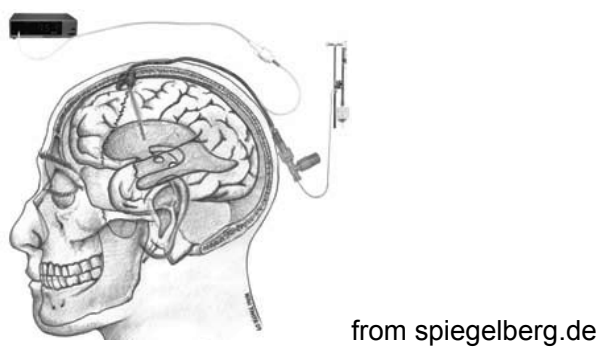


IOP: the details are not so important for the pressure measuring community: basic principle: deflection of the curvature of the cornea by applanation or air puff tonometry.

The lecture will also show a picture of the lymphatic system and the pressures there, just a little bit below the caval vein pressures.

Postmortem static vein pressure is normally around zero, when it has been a slow death. In case it would be around $15-20 \mathrm{~mm} \mathrm{Hg}$, then it was a fast death, not necessarily a crime, also an accident could be the reason.

\section{Pressure in medical devices, treating patients:}

Relevant for such pressure monitoring will be respratory pressures, pump head pressures in extracorporal circuits, infusion pumps, angiographic injectors, inflation devices.
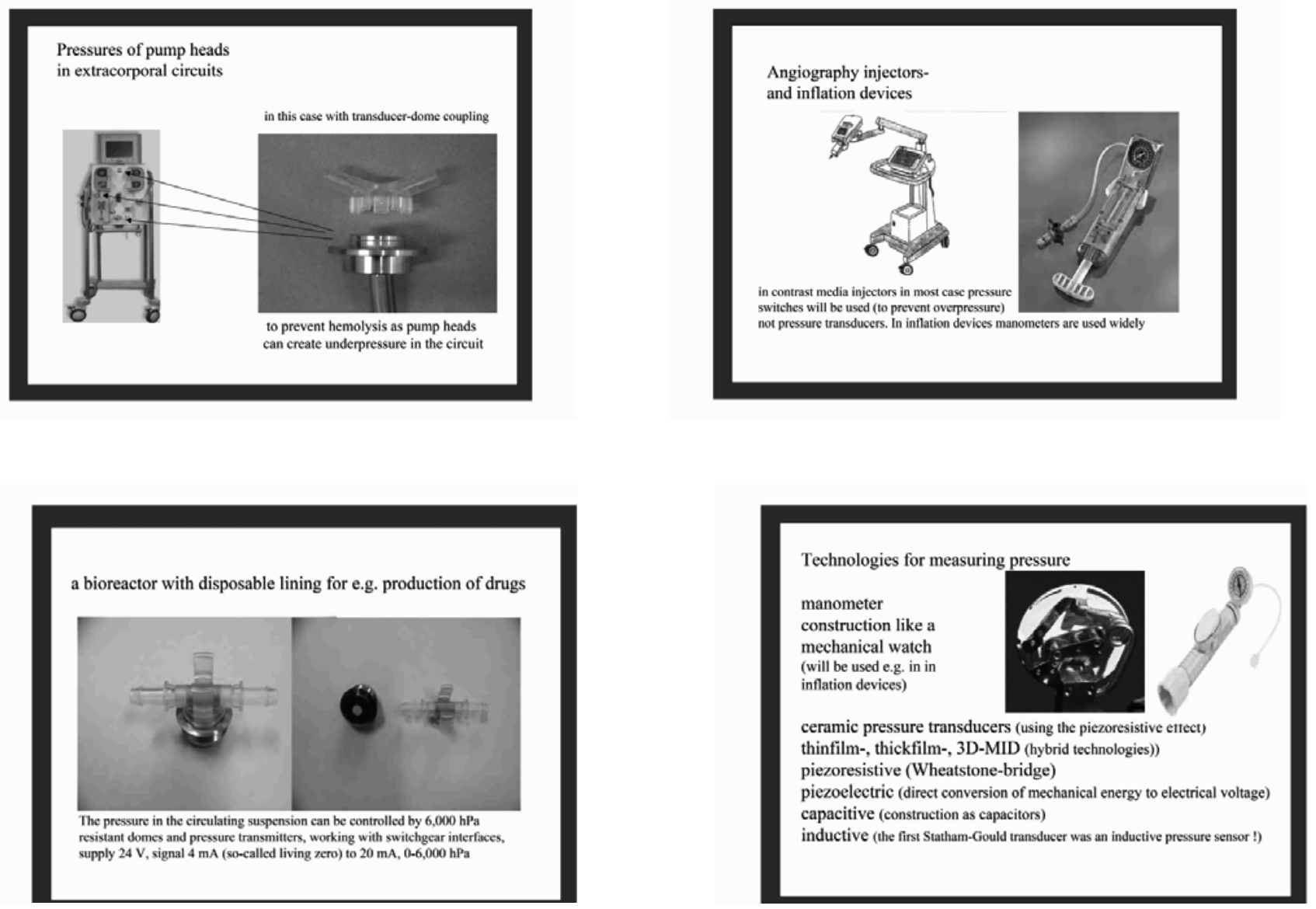

Even there is in the meantime a way to convert the manometer turn via the Hall effect to an electrical signal (see IntelliGauge from WIKA) the next displayed principles are the ones which really matter 


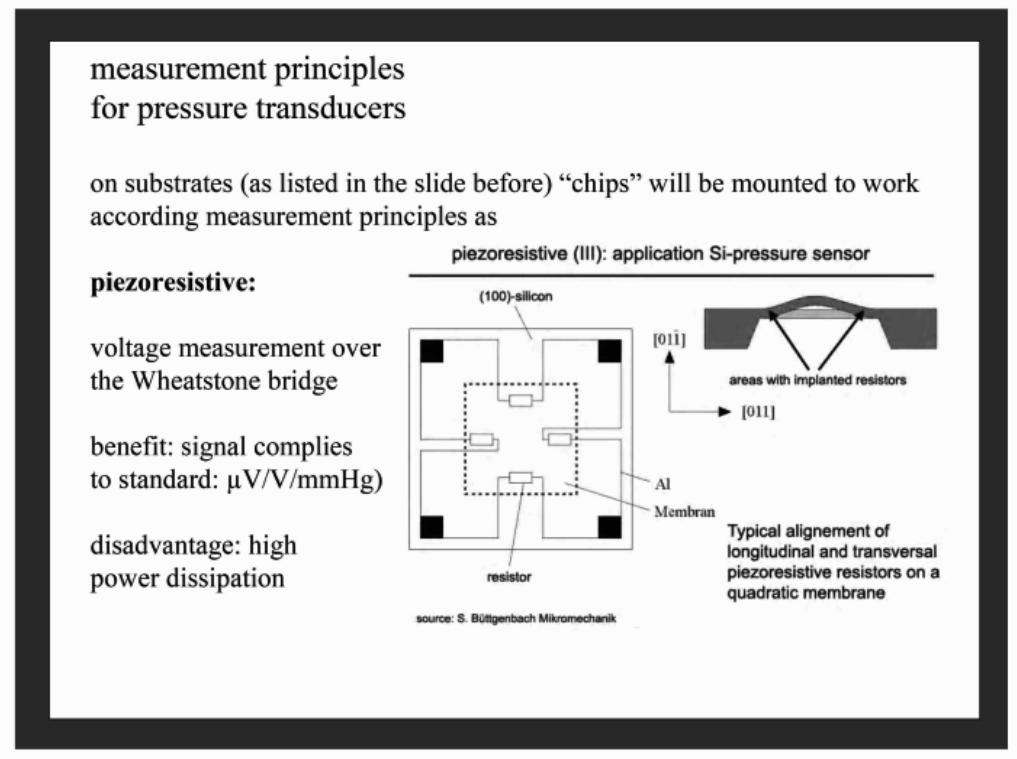

The capacitive pressure structur

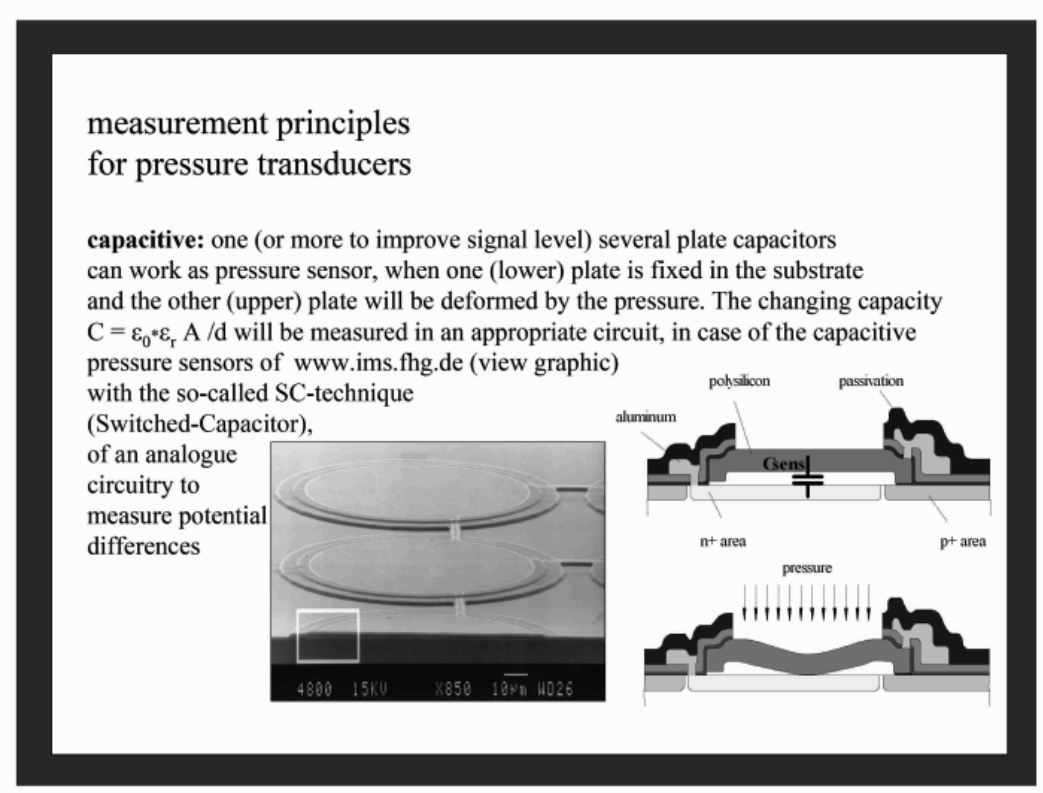

acc. to the ISO 60601-234 standard for blood pressure measurement, the signal output is $5 \mu \mathrm{V} / \mathrm{V} / \mathrm{mmHg}$, that means, when the patient monitor excites the pressure transducer with the Wheatstone bridge with $5 \mathrm{~V}$, the signal will be $25 \mu \mathrm{V} / \mathrm{mmHg}$, displayed at the patient monitor.
These capacitive structures do not suffer from hogh powr-loss due to heating the Wheatstone bridge, but the signal does not comply to the ISO-60601-2-34 standard, in case it shall, it has to be converted. Or a somehow different interface to the patient monitor is necessary.

In the lecture shortly also the piezoelectric and inductive measurement principles are discussed. 
a first capacitive pressure system which was implanted

ITES was a BMBF funded project around the year 2000 which

originally wanted to run a Siemens pressure chip in a telemetric set-up, without battery, but finally ended up in prototypes running with battery.

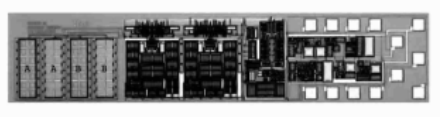

Drucksensor

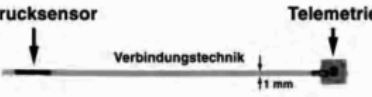

$2 \mathrm{~cm}$

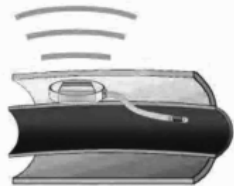

Pictures from the publication of B. Glasbrummel, G. Muhr in MEDRubin 2001,

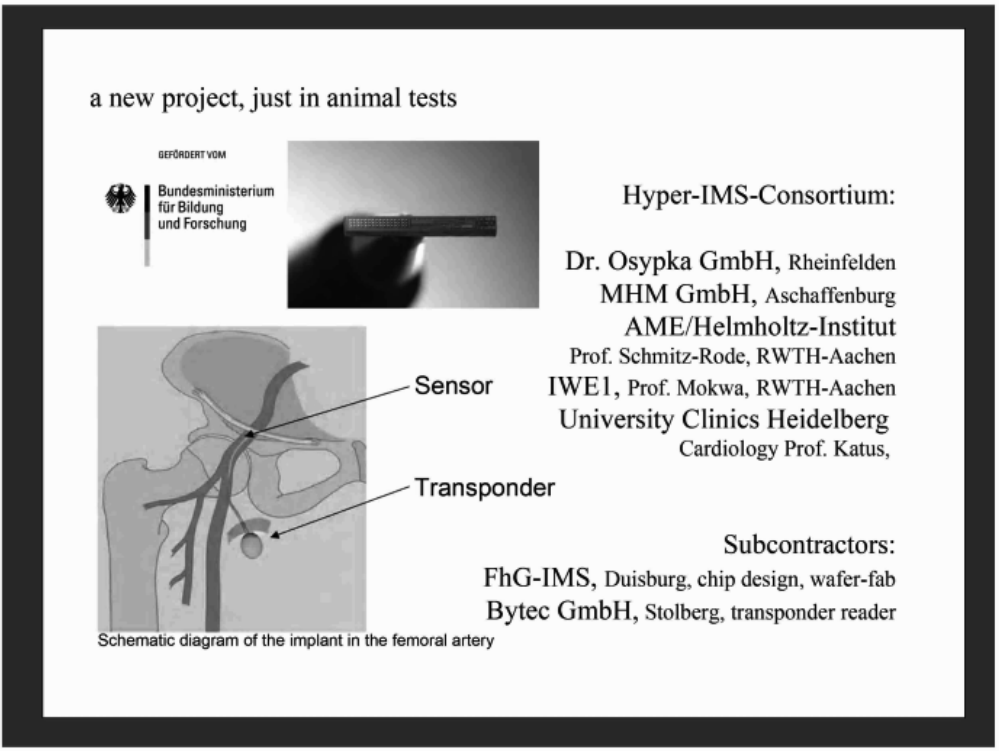

